\title{
HDAC6 inhibitor Cay10603 inhibits high glucose-induced oxidative stress, inflammation and apoptosis in retinal pigment epithelial cells via regulating NF- $\mathrm{KB}$ and NLRP3 inflammasome pathway
}

\author{
Qingsong Yang, Sizhen Li, Zixiu Zhou, Min Fu, Xiaodong Yang, Kuanxiao Hao and Yating Liu
}

Nanjing Tongren Eye Center, Nanjing Tongren Hosipital, Nanjing, Jiangsu, China

\begin{abstract}
The present study aimed to investigate the effects of histone deacetylase 6 (HDAC6) inhibitor Cay10603 (Cay) on high glucose (HG)-stimulated human retinal pigment epithelium (RPE) cells and its underlying mechanisms. ARPE-19 cells were cultured under normal glucose (NG) or high glucose (HG) conditions. The results revealed that HDAC6 was upregulated in HG-stimulated ARPE-19 cells. Cay treatment caused a decrease in intracellular reactive oxygen species (ROS). The levels of malondialdehyde (MDA) and myeloperoxidase (MPO) were reduced accompanied by increase in the activities of superoxide dismutase (SOD) and catalase (CAT) after treatment with Cay. Besides, Cay decreased the levels of tumor necrosis factor- $\alpha$ (TNF- $\alpha$ ), interleukin- $1 \beta$ (IL-1 $1 \beta$ ), IL- 6 and monocyte chemoattractant protein-1 (MCP-1) in supernatant. Meanwhile, the apoptotic rate in Cay-treated ARPE-19 cells notably reduced, coupled with an upregulation in Bcl-2 expression and a downregulation in cleaved caspase-3 and cleaved caspase-9 expression. Cay decreased the expression of phospho (p)-NF-кB p65, p-IкB- $\alpha$, NLRP3, cleaved caspase-1 and ASC while increased the expression of NF- $\mathrm{kB}$ p65 (cytoplasm). Taken together, these findings demonstrated that Cay suppressed HG-induced oxidative stress, inflammation and apoptosis via regulating NF- $\mathrm{kB}$ and NLRP3 inflammasome pathway in HG-induced ARPE-19 cells, suggesting that Cay might be a therapeutic agent for the treatment of diabetic retinopathy.
\end{abstract}

Key word: HDAC6 - Inflammatory response - Apoptosis - Bcl-2 - Reactive oxygen species

\section{Introduction}

Diabetic retinopathy (DR), a kind of the most common and prevalent microvascular complications of diabetes, remains a major cause of vision loss among working-age adults (Hendrick et al. 2015). It is well known that vision loss is closely implicated in the damage of retinal pigment epithelium (RPE) cells which serve as a critical component of the outer blood-retina barrier (Xia and Rizzolo 2017; Chen et al. 2019). Excessive circulating level of glucose is the dominant characteristic of diabetes patients, which makes RPE cells undergo oxidative stress, inflammation response and apoptosis and eventually results in the damage of visual function (Farnoodian et al. 2016; Li et al. 2017). At present, there is no effective therapeutic agent to inhibit high glucose

Correspondence to: Qingsong Yang, Nanjing Tongren Hosipital, NO. 2007, Jiyin Avenue, Jiangning District, Nanjing, Jiangsu, 211102, China

E-mail: qiangsongshine@163.com
(HG)-induced damage. Therefore, it is of utmost importance to understanding the underlying mechanisms and developing effective methods to treat DR.

A growing body of evidence suggested that oxidative stress and inflammation play significant roles in the occurrence and development of DR (Adamis 2002; Kastelan et al. 2013; Rangasamy et al. 2014). The excess reactive oxygen species (ROS) leads to the secretion of pro-inflammatory cytokines including tumor necrosis factor- $\alpha$ (TNF- $\alpha$ ), interleukin (IL)- 6 and IL-1 $\beta$. Importantly, excessive accumulation of ROS contributes to the damage of macromolecules and leads to cell death through apoptosis of RPE cells (Shi et al. 2019b). Hence, inhibition of oxidative stress, inflammation and apoptosis might help to cure DR in clinic.

Histone deacetylase 6 (HDAC6) is structurally and functionally unique, and plays multiple of biological roles via deacetylation of a variety of target molecules (Li et al. 2013). It has been well reported that HDAC inhibitor Tubastatin-A can restore RPE function in hyperglycemia (Desjardins et al. 2016). Report has demonstrated previously that TDP-43 
could protect retinal ganglion cells against oxidative stress and apoptosis via restraining HDAC6 expression (Yi et al. 2019). However, the effect of Cay 10603 (Cay), a novel inhibitor of HDAC6, in DR has not been addressed.

In the current study, HG was used to induce human RPE cell line ARPE-19 to mimic DR model in vitro. Then, Cay was applied to treat with cells and the mechanisms were investigated.

\section{Materials and Methods}

\section{Cell culture and treatment}

The human RPE cell line ARPE-19 was purchased from the American Type Culture Collection (ATCC, Manassas, VA, USA). Cells were cultured in DMEM-F12 medium (Hyclone, Logan, UT, USA) supplemented with $10 \%$ fetal bovine serum (FBS; Gibco, USA) in a humidified incubator at $37^{\circ} \mathrm{C}$ containing $5 \% \mathrm{CO}_{2}$. Cells were maintained in medium supplemented with normal glucose (NG, $5.5 \mathrm{mM}$ glucose), $5.5 \mathrm{mM}$ glucose $+22.5 \mathrm{mM}$ mannitol (osmotic pressure control, OP) and HG (25 mM glucose) for 48 h. Cay10603 (Cay), a new HDAC6 inhibitor, was acquired from Selleck Chemicals (Houston, Texas). ARPE-19 cells were pretreated with Cay for $2 \mathrm{~h}$ before exposure to HG condition.

\section{Cell Counting Kit-8 (CCK-8) assay}

The capacity of cell proliferation was evaluated by CCK-8 assay (Shanghai Yi Sheng Biotechnology Co., Ltd., Shanghai, China). ARPE- 19 cells $\left(1 \times 10^{4}\right.$ cells per well) were plated in a 96 well plate. Cells were treated with a series of concentrations of Cay $(1,2.5,5$ and $10 \mu \mathrm{M})$ at $37^{\circ} \mathrm{C}$ in an atmosphere containing $5 \% \mathrm{CO}_{2}$. After incubation with Cay for 24 and $48 \mathrm{~h}, 10 \mu \mathrm{l}$ CCK- 8 solution was added into the microplate. Following incubation for $1 \mathrm{~h}$, absorbance at $450 \mathrm{~nm}$ was detected using a microplate reader.

\section{Evaluation of oxidative stress}

To evaluate the levels of oxidative stress, oxidative stress biomarkers including the concentration of malondialdehyde (MDA) as well as the activities of myeloperoxidase (MPO), superoxide dismutase (SOD) and catalase (CAT) were detected by commercial kits according to the colorimetric methods following the manufacturer's guidelines. Above kits were all provided by Nanjing Jiancheng Bioengineering Institute (Nanjing, China).

\section{Enzyme-linked immunosorbent assay (ELISA) assay}

The concentrations of inflammatory factors including TNF- $\alpha$, IL- $1 \beta$, IL- 6 and monocyte chemoattractant protein-1
(MCP-1) in culture medium samples were examined by commercially available ELISA Kit (Shanghai Xitang Biotechnology Co., Ltd., Shanghai, China).

\section{Measurement of intracellular ROS}

The production of intracellular ROS was measured with an ROS assay kit (Beyotime Biotechnology, Shanghai, China) using 2'7'-dichlorodihydrofluorescein diacetate (DCFHDA) as a fluorescence probe. Cells were seeded into 96-well plate with the density of $2 \times 10^{4}$ cells per well. After treatment with Cay and exposure in medium containing HG $(25 \mathrm{mM})$, ARPE-19 cells were incubated with DCFH-DA $(10 \mu \mathrm{M})$ in the dark. The fluorescence released was detected using a fluorimeter at an excitation and emission wavelength of $480 \mathrm{~nm}$ and $520 \mathrm{~nm}$. A confocal microscope was employed to obtain the images.

\section{Cell apoptosis assay}

Cell apoptosis assay was performed using flow cytometry. Briefly, ARPE-19 cells were pretreated with Cay for $2 \mathrm{~h}$, followed by incubation in HG $(25 \mathrm{mM})$ condition for $48 \mathrm{~h}$. Cells were suspended in Binding Buffer and stained with Annexin V-FITC and propidium iodide (PI) using a cell apoptosis detection kit (KeyGEN, Nanjing, China). Then, cell apoptosis was analyzed by a flow cytometry (BD Biosciences, NJ, USA). The percentage of apoptotic cells was analyzed using FlowJo software (Becton-Dickinson-San Jose CA, USA).

Reverse transcription-quantitative polymerase chain reaction (RT-qPCR) analysis

Total RNA was extracted from cells using Trizol reagent (Invitrogen). Then, first-strand cDNA was synthesized from RNA using a Sensiscript RT kit (Takara Biotechnology Co., Ltd., Japan) following the manufacturer's recommendations. Then, qPCR was conducted with SYBR Green Supermix (Bio-Rad Laboratories, Inc.) on the ABI 7500 system (Applied Biosystems; Thermo Fisher Scientific, Inc.). Relative gene expression was normalized to GAPDH.

\section{Western blotting}

Total proteins were extracted using RIPA lysis buffer (Beyotime, Shanghai, China). Then, a BCA Kit (Thermo Fisher Scientific, Paisley, UK) was executed for detecting the concentrations of proteins. A total of $40 \mu \mathrm{g}$ protein samples in cell lysates were separate in 10\% SDS-PAGE gels, followed by transferring to polyvinylidene difluoride membranes (Millipore, Bedford, MA). The unspecific bands were blocked with $5 \%$ fat-free milk. Subsequently, these membranes were probed with primary antibodies. Following washing with 
TBST for three times, these membranes were incubated with horseradish peroxidase-conjugated secondary antibody (Abcam, Cambridge, UK). The bands were visualized using an Odyssey Infrared Imaging Scanner (LI-COR Biosciences). Intensity of bands were examined using Image-J software (National Institutes of Health, Bethesda, MA, USA). The protein expression was normalized to GAPDH levels.

\section{Statistical analysis}

All experiments were performed in triplicate. The data are expressed as mean \pm SD. All statistical analyses were made using SPSS 22.0 software (Chicago, IL). Comparisons among different groups were analyzed by Student's $t$-test or oneway analysis of variance (ANOVA). A value of $p<0.05$ was considered statistically significant.

\section{Results}

\section{HDAC6 was highly expressed in HG-induced ARPE-19 cells}

To investigate the function of HDAC6 in ARPE-19 cells induced by HG, the expression of HDAC6 was examined by Western blotting and RT-qPCR, respectively. As presented in Figure 1A, the expression of HDAC6 in osmotic pressure control presented no obvious changes compared with NG group. After HG induction, significantly upregulated HDAC6 protein expression was observed. Concurrently, the mRNA expression level of HDAC6 determined by RTqPCR was in accordance with the above (Fig. 1B). These date indicated that the expression of HDAC6 was upregulated in HG-induced ARPE-19 cells.

Cay treatment notably enhanced the capacity of cell proliferation in HG-induced ARPE-19 cells

The effect of Cay on cell proliferation was detected in this study. After treatment with different doses of Cay $(1,2.5$, 5 and $10 \mu \mathrm{M}$ ) for $48 \mathrm{~h}, \mathrm{CCK}-8$ assay was applied for detect cell viability. From the result of Figure 1C, we found that the administration of $10 \mu \mathrm{M}$ Cay presented a remarkably effect on the viability of ARPE-19 cells whereas cell viability had no obvious changes after treatment with Cay at doses of 1, 2.5 and $5 \mu \mathrm{M}$. Then, the effect of Cay on proliferation of ARPE-19 cells induced by HG was investigated. As exhibited in Figure 1D, HG treatment notably decreased the cell viability in HG-stimulated ARPE-19 cells. However, Cay treatment alleviated the inhibitory effect of HG stimulation on the ARPE-19 cells viability in
A

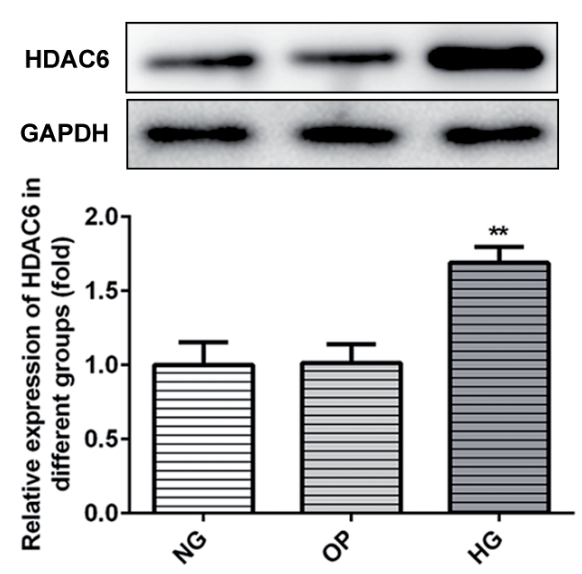

C

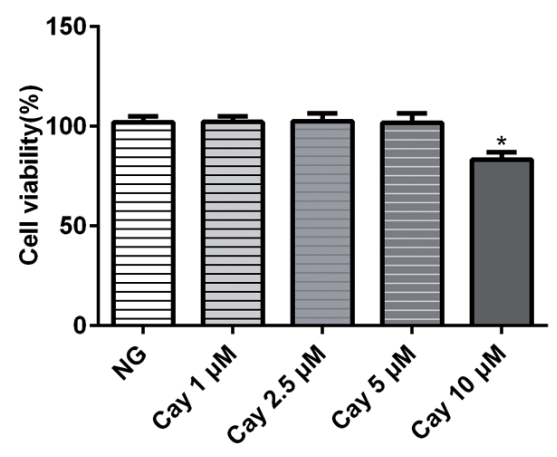

B

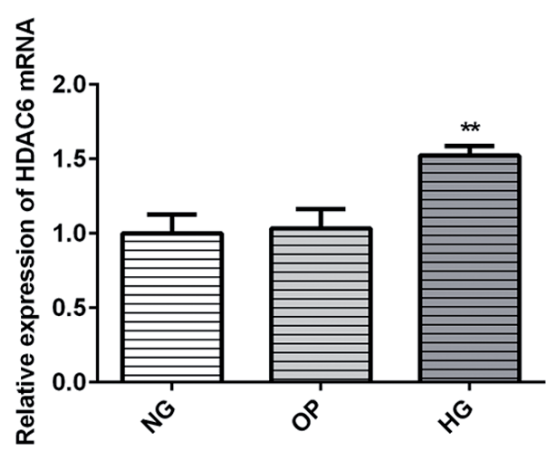

Figure 1. HDAC6 was highly expressed in $\mathrm{HG}$-induced ARPE- 19 cells and Cay treatment increased the capacity of cell proliferation in HGinduced ARPE-19 cells. The expression of HDAC6 was assessed by Western blotting (A) and RT-qPCR (B). C. The viability of ARPE-19 cells was determined by Cell Counting Kit-8 (CCK-8) assay after cells being treated with a series of concentrations of Cay $(1,2.5,5$ and $10 \mu \mathrm{M})$ for 48 h. D. Cell viability was measured using CCK-8 assay in HG-induced ARPE-19 cells after pretreatment with Cay. ${ }^{*} p<0.05 v s$. NG group; ${ }^{\star *} p<0.01 v s$. OP group; ${ }^{*} p<$ $0.05,{ }^{\# \#} p<0.01$ vs. HG group. HDAC6, histone deacetylase 6; OP, osmotic pressure control; HG, high glucose; NG, normal glucose; Cay, Cay10603. 

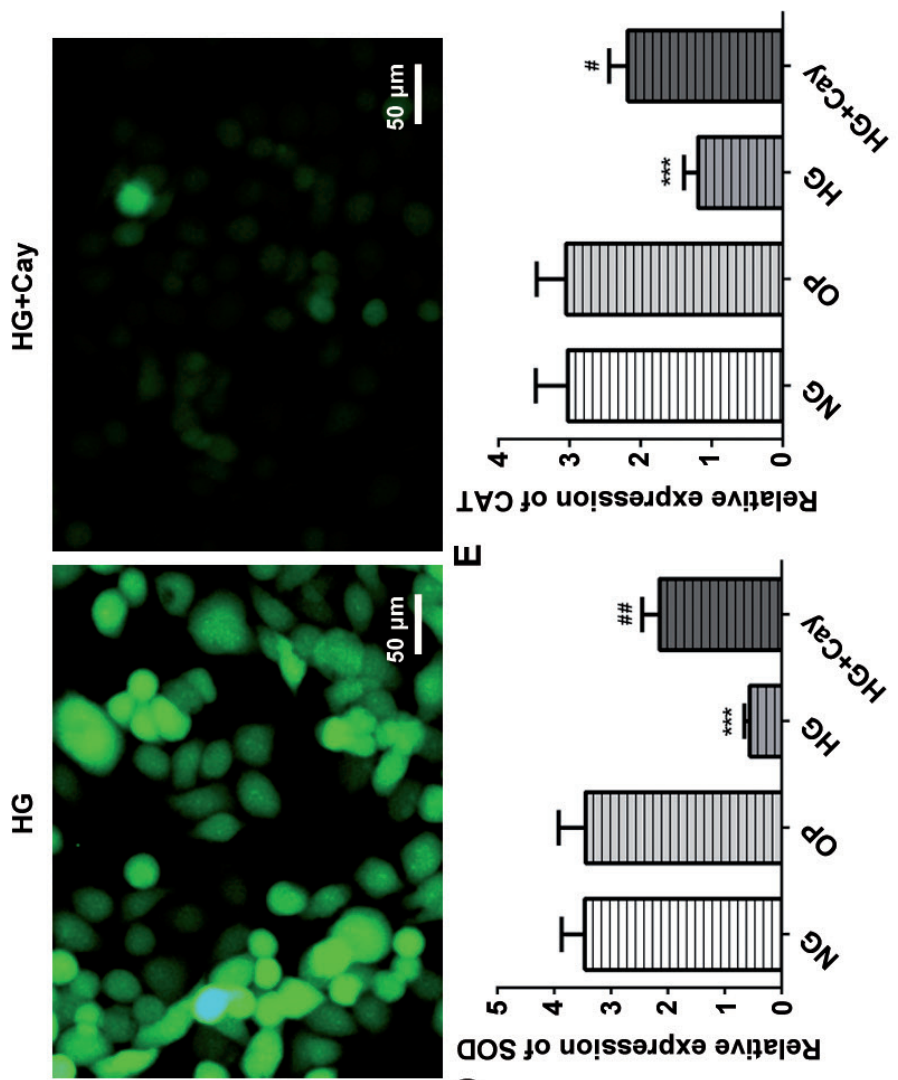

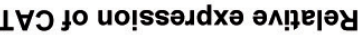
ш

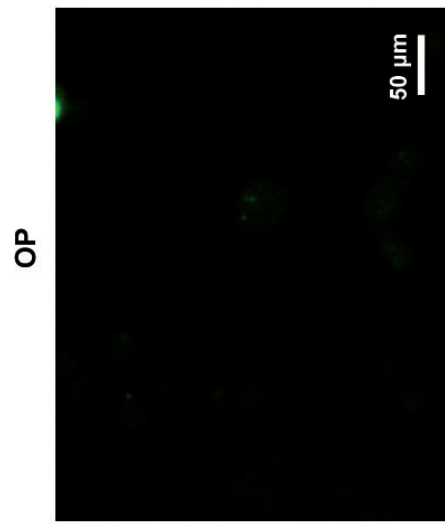

0
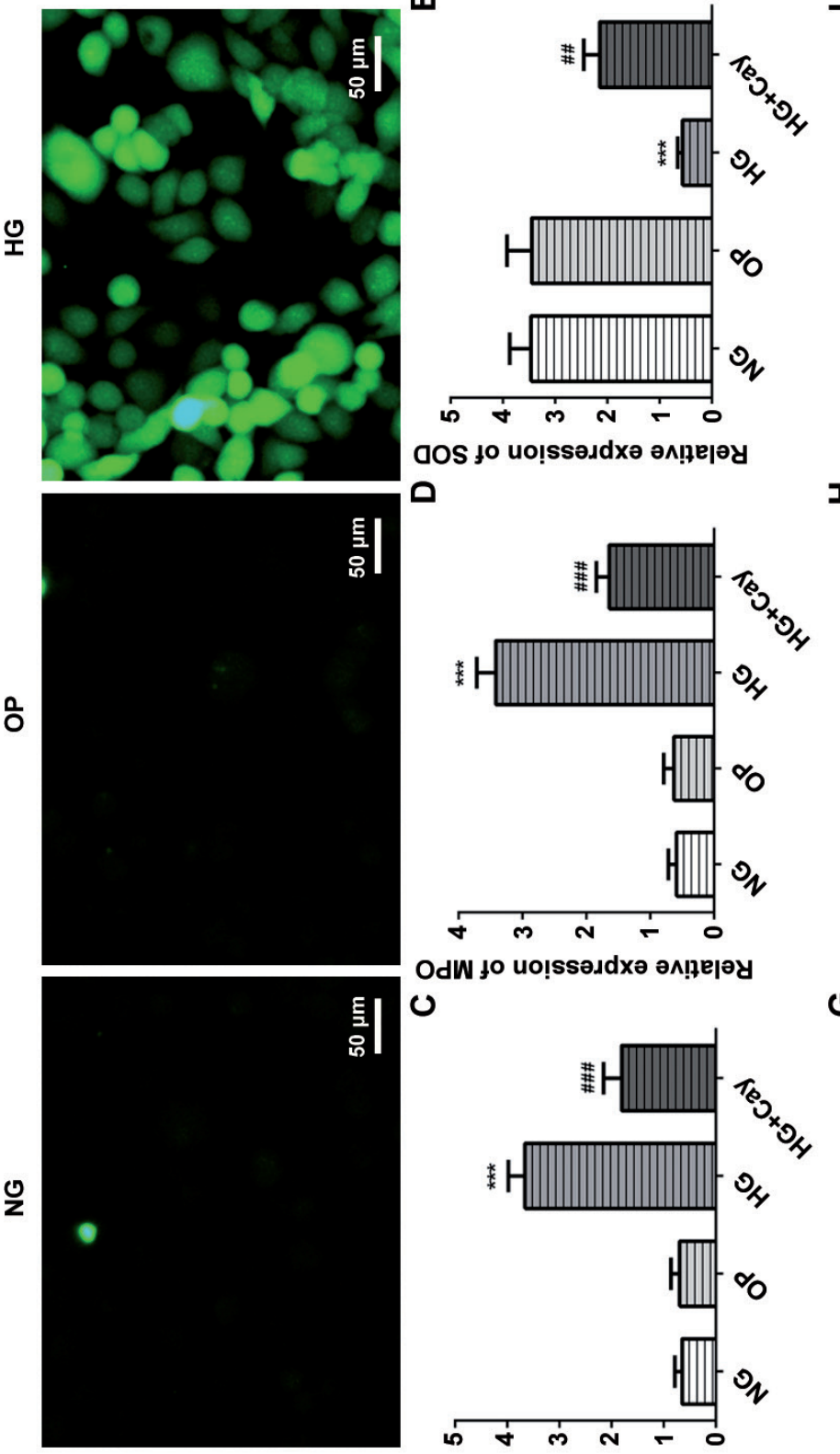

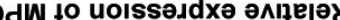

0

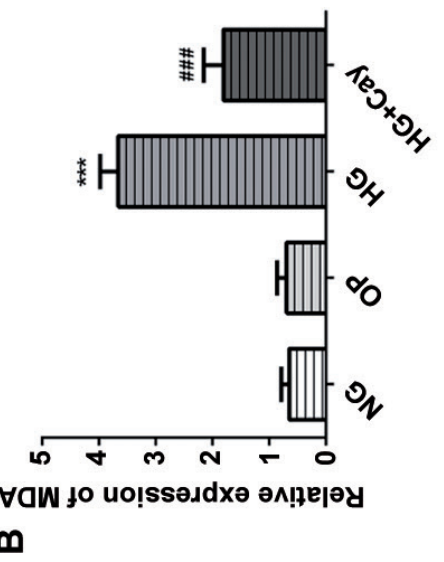

(

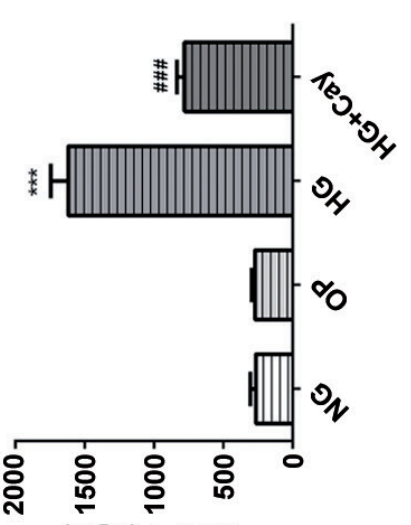

苞我官

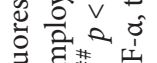

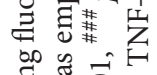

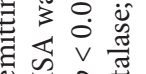

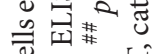

过论

0 bo $v \dot{v}$

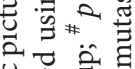

(7/6u) L-dow

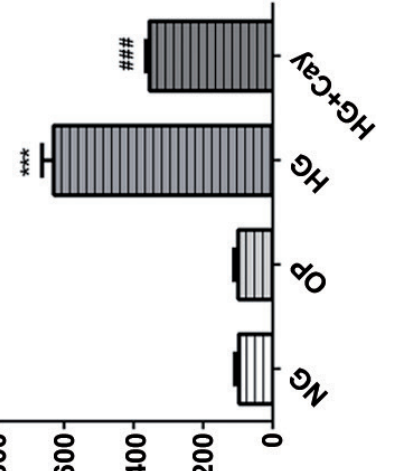

政

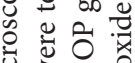

光藏它

这

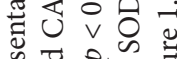

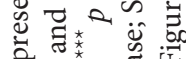

I

(7/6u) 9-7

$\approx \widehat{\theta} \dot{\vec{\theta}}$

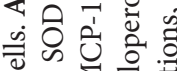

बें

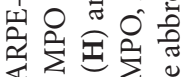

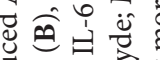

藏昰

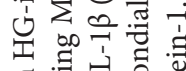

$\exists \exists \exists$

跣

额崖安

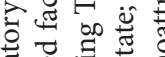

离 离离

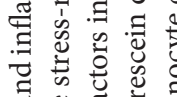

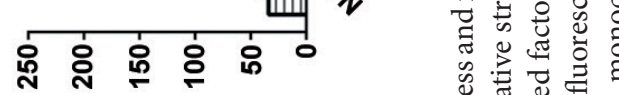

(7/6u) dเ-7

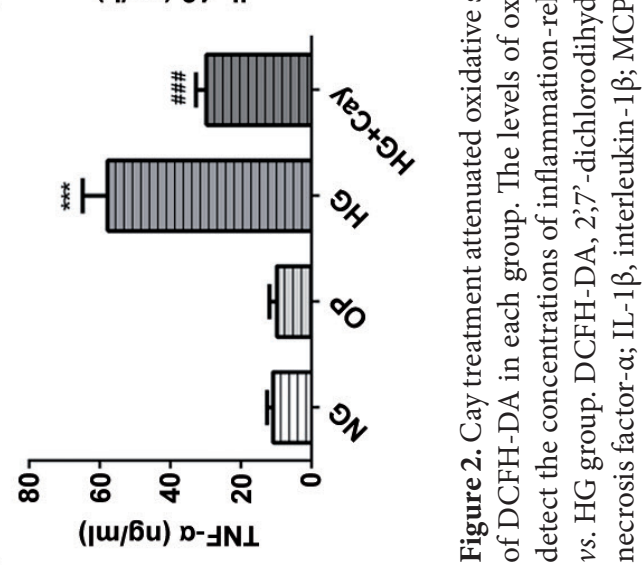



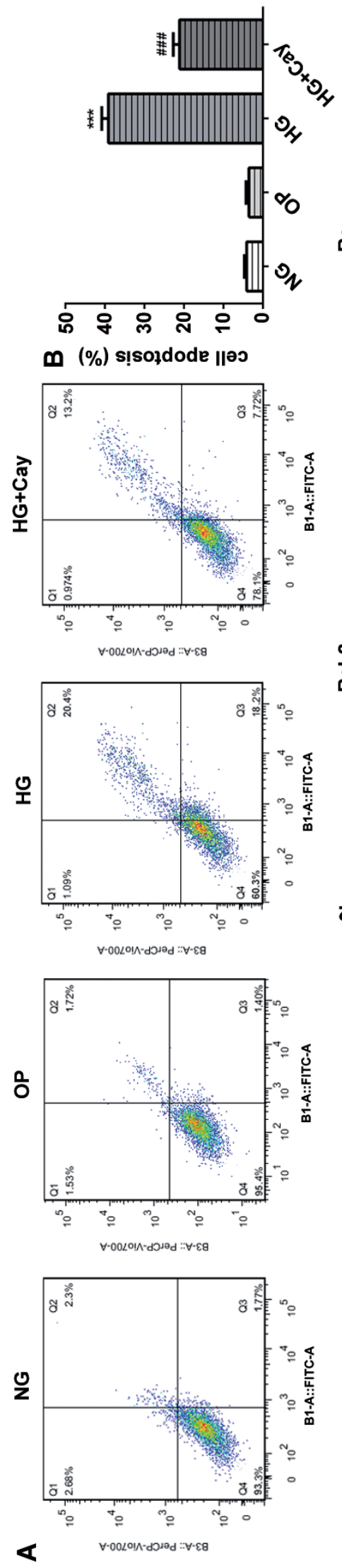

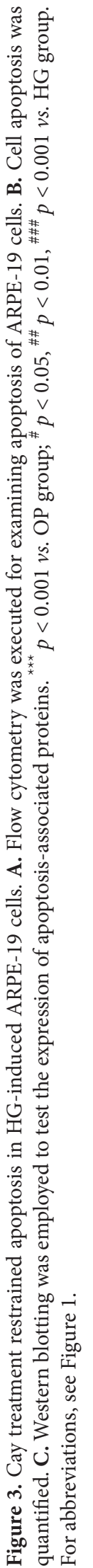




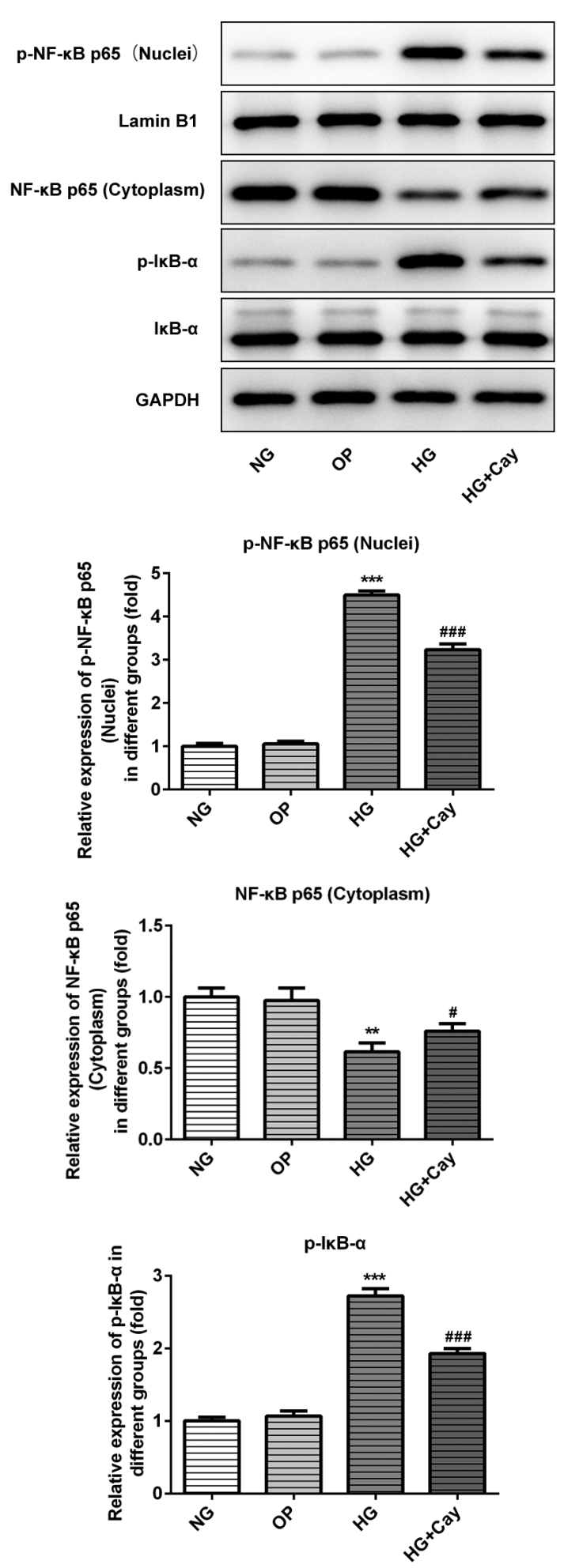

Figure 4. Cay treatment suppressed NF- $\mathrm{B}$ signaling in HGinduced ARPE-19 cells. The expression of p-NF- $\kappa \mathrm{B}$ p65 (nulei),

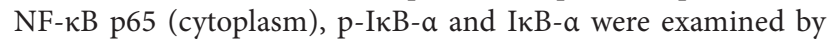
Western blotting. ${ }^{* *} p<0.01,{ }^{* * *} p<0.001$ vs. OP group; ${ }^{\#} p<0.05$, \#\#\# $p<0.001 v$ s. HG group. NF- $\kappa \mathrm{B}$, nuclear factor kappa B; $\mathrm{p}-\mathrm{NF}-\kappa \mathrm{B}$ p65, phospho-NF- $\kappa$ B p65. For more abbreviations, see Figure 1. a dose-dependent manner. Therefore, Cay $5 \mu \mathrm{M}$ was used in the following experiments.

Cay treatment attenuated oxidative stress in HG-induced ARPE-19 cells

To confirm the effect of HDAC6 inhibitor Cay on oxidative stress induced by HG stimulation, the production of intracellular ROS was measured by DCFH-DA assay. As presented in Figure 2A, HG induction caused a significant increase in the generation of ROS compared with OP group, whereas obvious decrease of that of was observed after treatment with Cay. Meanwhile, the levels of oxidative stress-related factors were examined by kits. We found that the levels of MDA and MPO was reduced significantly accompanied by an evident increase in the activities of SOD and CAT after ARPE-19 cells being stimulated by HG (Fig. 2B-E). The effects of HG on above factors were alleviated following treatment with Cay. Overall, these findings demonstrated that Cay treatment mitigated HG-induced oxidative stress in ARPE-19 cells.

Cay treatment relieved inflammatory response in $H G$-induced ARPE-19 cells

In order to explore the effect of Cay on inflammation induced by HG in ARPE-19 cells, the concentrations of inflammation-related cytokines were detected by ELISA. As shown in Figure 2F-I, HG induction remarkably increased the levels of TNF- $\alpha$, IL- $1 \beta$, IL- 6 and MCP- 1 in comparison with OP group. After intervention with Cay, the concentrations of above inflammatory factors were dramatically decreased relative to $\mathrm{HG}$ group. These observations revealed that HDAC6 inhibitor Cay could alleviate HG-induced inflammatory response in ARPE-19 cells.

Cay treatment inhibited apoptosis in HG-induced ARPE-19 cells

Flow cytometry was executed for examining the apoptosis of ARPE-19 cells induced by HG and the result was demonstrated in Figure 3A and B. We found that the number of apoptosis ARPE-19 cells was obviously increased after stimulation with HG. Importantly, Cay treatment led to a notably decrease in the rate of apoptosis cells relative to HG induction alone. Concurrently, the expression of apoptosis-related proteins were evaluated by Western blotting. As presented in Figure 3C, HG stimulation downregulated the expression of Bcl-2 coupled with an upregulation in Bax, cleaved caspase- 3 and cleaved caspase- 9 expression in comparison with OP group. Then, the changes in expressions the $\mathrm{Bcl}-2$, Bax, cleaved caspase- 3 and cleaved caspase- 9 caused by HG stimulation were reversed after intervention with Cay. These 
data suggested that Cay treatment inhibited apoptosis in HG-induced ARPE-19 cells.

Cay treatment suppressed NF- $\kappa B$ and NLRP3 inflammasome pathway in HG-induced ARPE-19 cells

To determine the underlying mechanisms of Cay in HGinduced ARPE-19 cells, the NF- $\kappa$ B and NLRP3 inflammasome pathway was investigated in this study. Western blot analysis proved that HG stimulation enhanced the expres-

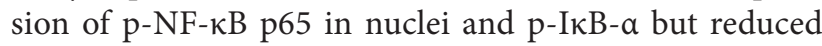
the expression of NF- $\kappa B$ p65 in cytoplasm (Fig. 4). At the same time, the expression of NLRP3 inflammasome-related proteins including NLRP3, cleaved caspase-1 and apoptosisassociated speck-like protein (ASC) were increased in HG group compared with OP group (Fig. 4 and 5). Following treatment with Cay, the effects of HG stimulation on the expression of above proteins were reversed. Taken together, these findings confirmed that Cay treatment suppressed NF- $\kappa \mathrm{B}$ and NLRP3 inflammasome pathway in HG-induced ARPE-19 cells.

\section{Discussion}

In the present study, when we simulated diabetic environment under HG condition in ARPE-19, the expression of HDAC6 was markedly upregulated. And treatment with HDAC6 inhibitor Cay suppressed oxidative stress, inflammation and apoptosis induced by HG stimulation via regulating the NF- $\kappa \mathrm{B}$ and NLRP3 inflammasome pathway, suggesting that Cay may possess therapeutic value in the treatment of DR.

Numerous studies unveil that diabetes associated hyperglycaemia can lead to a rise in ROS production, which is considered as a major causative factor in DR development (Calderon et al. 2017; Li et al. 2017). Persistent hyperglycemia induces the generation of ROS and triggers oxidative stress. And clinical evidence have clearly confirmed that oxidative stress plays a crucial role in the pathogenesis of DR (Wu et al. 2014; Kowluru et al. 2015). The presence of oxidative stress increased the levels of lipid peroxidation end products MDA and MPO whereas decreased the activities of antioxidant enzyme including SOD and CAT. Additionally, oxidative stress serves as a trigger in diabetic retina, which can trigger the secretion of pro-inflammatory cytokines and the expression of genes related to inflammatory cytokines (Al-Kharashi 2018; Lv et al. 2019). Previous studies have highlighted the importance of HDAC6 inhibition in regulating oxidative stress and inflammation in a variety of diseases, especially in DR (Shi et al. 2017; Yi et al. 2019; Zhang et al. 2019a). The effect of Cay, a novel inhibitor of HDAC6, was investigated in this research, and we demonstrated that Cay treatment
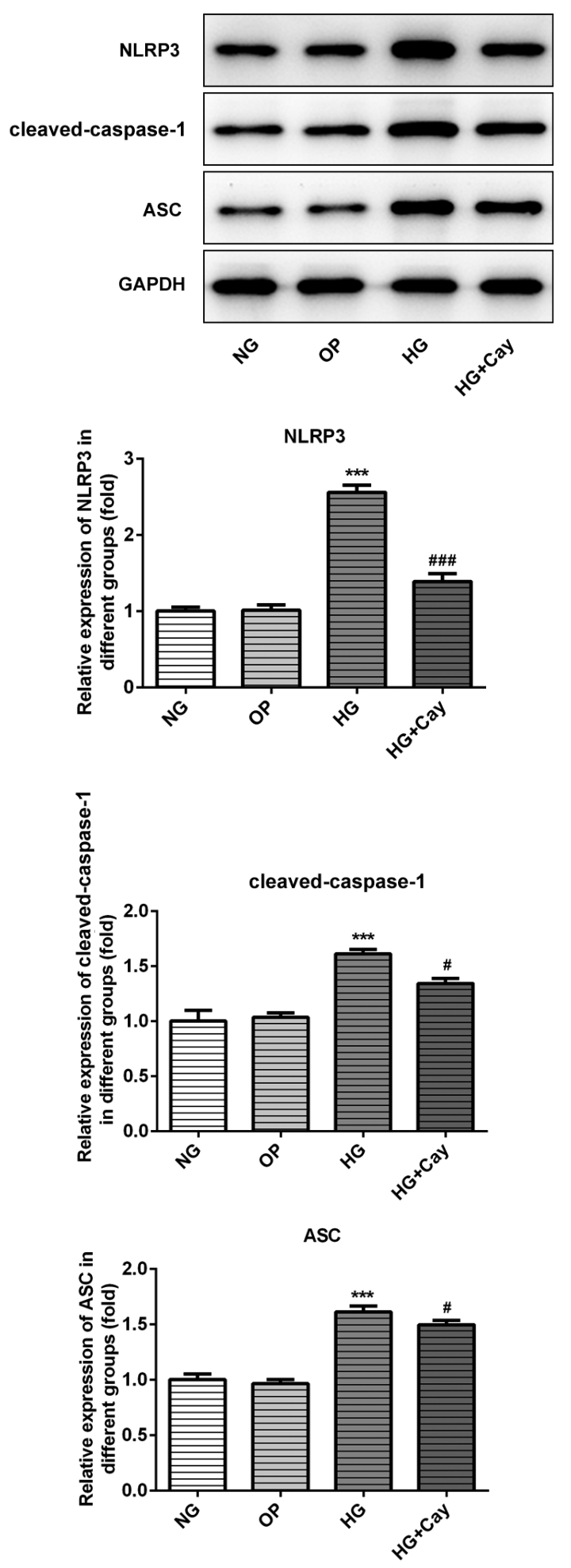

Figure 5. Cay treatment inhibited NLRP3 inflammasome pathway in HG-induced ARPE-19 cells. The expression of NLRP3, caspase-1 and ASC were evaluated by Western blotting. ${ }^{* * *} p<$ 0.001 vs. OP group; ${ }^{\#} p<0.05,{ }^{\# \# \#} p<0.001 v s$. HG group. ASC, apoptosis-associated speck-like protein. For more abbreviations, see Figure 1. 
inhibited HG-induced oxidative stress and inflammatory responses in ARPE-19 cells.

It is also worth noting that robust evidence suggests that sustained hyperglycemia triggers apoptosis in RPE cells, which is a major pathological change in the eyes of patients with DR (Willermain et al. 2018; Yu et al. 2019; Zhang et al. 2019b). Numerous studies unveil that inhibition of apoptosis of RPE cells contributes to protect against DR (Jiang et al. 2017; Yu et al. 2018). It has been well reported that HDAC6 inhibition provides intestinal protection via decreasing apoptotic responses during hemorrhagic shock (Chang et al. 2019). TDP-43 could protects retinal ganglion cells against oxidative stress and apoptosis via restraining HDAC6 expression (Yi et al. 2019). In this study, HG stimulation promoted apoptosis of RPE cells, which was observed by an increase in apoptosis rate, accompanied by downregulated Bcl-2 expression and upregulated Bax, cleaved caspase- 3 and cleaved caspase- 9 expression. Above results were in agreement with the previous findings (Shi et al. 2019a; Zhou et al. 2019). Importantly, treatment with Cay decreased HG-induced apoptosis. These results presented the anti-apoptotic potential of Cay in DR treatment.

Mounting evidence supported the view that nuclear factor $\mathrm{NF}-\kappa \mathrm{B}$ is a transcription factor with multiple regulatory functions in cells (Xia et al. 2014). Reports have demonstrated previously that the activity of NF- $\kappa \mathrm{B}$ is closely related to $\mathrm{DR}$ and inhibition of NF- $\kappa \mathrm{B}$ signaling can protect against HG-induced injury of RPE cells (Xiao et al. 2019; Zhang et al. 2019c). In addition, NLRP3 inflammasome pathway was reported to participate in the progress of DR (Hao et al. 2019). Inactivation of NLRP3 inflammasome pathway contributes to recover from HG-induced oxidative stress, inflammation and apoptosis (Lu et al. 2018; Li et al. 2019). Our results indicate that Cay treatment notably decreased the expression of NF- $\kappa$ B p 65 in nuclei and $\mathrm{p}-\mathrm{I} \kappa \mathrm{B}-\alpha$ while increased the expression of NF- $\kappa \mathrm{B}$ p 65 in cytoplasm. Concurrently, the expression of NLRP3, cleaved-caspase-1 and ASC were downregulated following treatment with Cay. Overall, these observations reveal that Cay protects against HG-induced RPE cells injury via inhibiting NF- $\kappa \mathrm{B}$ and NLRP3 inflammasome pathway.

\section{Conclusion}

In summary, these investigations for the first time delineated that HDAC6 inhibitor Cay could restrain oxidative stress, inflammation and apoptosis in human RPE cells induced by $\mathrm{HG}$ via regulating the NF- $\kappa \mathrm{B}$ and inflammasome pathway. These findings corroborated that Cay may possess therapeutic value in the treatment of DR, which provides experimental and scientific evidences for the potential of using Cay as a therapeutic agent in clinical DR patients.
Acknowledgements. Not applicable.

Conflict of interest. The authors declare no conflict of interest.

\section{References}

Adamis AP (2002): Is diabetic retinopathy an inflammatory disease? Br. J. Ophthalmol. 86, 363-365 https://doi.org/10.1136/bjo.86.4.363

Al-Kharashi AS (2018): Role of oxidative stress, inflammation, hypoxia and angiogenesis in the development of diabetic retinopathy. Saudi. J. Ophthalmol. 32, 318-323 https://doi.org/10.1016/j.sjopt.2018.05.002

Calderon GD, Juarez OH, Hernandez GE, Punzo SM, De la Cruz ZD (2017): Oxidative stress and diabetic retinopathy: development and treatment. Eye (Lond) 31, 1122-1130 https://doi.org/10.1038/eye.2017.64

Chang PP, Bhatti UF, Williams AM, Dennahy IS, Liu BL, Li YQ, Alam HB (2019): Inhibition of histone deacetylase 6 attenuates intestinal inflammation and apoptosis in a rodent model of hemorrhagic shock. J. Trauma. Acute Care Surg. 86, 874-880 https://doi.org/10.1097/TA.0000000000002169

Chen P, Miao Y, Yan P, Wang XJ, Jiang C, Lei Y (2019): MiR-455-5p ameliorates HG-induced apoptosis, oxidative stress and inflammatory via targeting SOCS3 in retinal pigment epithelial cells. J. Cell. Physiol. 234, 21915-21924 https://doi.org/10.1002/jcp.28755

Desjardins D, Liu YY, Crosson CE, Ablonczy Z (2016): Histone deacetylase inhibition restores retinal pigment epithelium function in hyperglycemia. PloS One 11, 16 https://doi.org/10.1371/journal.pone.0162596

Farnoodian M, Halbach C, Slinger C, Pattnaik BR, Sorenson CM, Sheibani N (2016): High glucose promotes the migration of retinal pigment epithelial cells through increased oxidative stress and PEDF expression. Am. J. Physiol. Cell. Physiol. 311, C418-436

https://doi.org/10.1152/ajpcell.00001.2016

Hao JF, Zhang HK, Yu JB, Chen X, Yang L (2019): Methylene blue attenuates diabetic retinopathy by inhibiting NLRP3 inflammasome activation in STZ-induced diabetic rats. Ocul. Immunol. Inflamm. 27, 836-843 https://doi.org/10.1080/09273948.2018.1450516

Hendrick AM, Gibson MV, Kulshreshtha A (2015): Diabetic retinopathy. Prim. Care 42, 451-464 https://doi.org/10.1016/j.pop.2015.05.005

Jiang YY, Sang YZ, Qiu QH (2017): microRNA-383 mediates high glucose-induced oxidative stress and apoptosis in retinal pigment epithelial cells by repressing peroxiredoxin 3. Am. J. Transl. Res. 9, 2374-2383

Kastelan S, Tomic M, Gverovic Antunica A, Salopek Rabatic J, Ljubic S (2013): Inflammation and pharmacological treatment in diabetic retinopathy. Mediators Inflamm. 2013, 213130 https://doi.org/10.1155/2013/213130

Kowluru RA, Kowluru A, Mishra M, Kumar B (2015): Oxidative stress and epigenetic modifications in the pathogenesis of diabetic retinopathy. Prog. Retin. Eye Res. 48, 40-61 https://doi.org/10.1016/j.preteyeres.2015.05.001 
Li C, Miao X, Li F, Wang S, Liu Q, Wang Y, Sun J (2017): Oxidative stress-related mechanisms and antioxidant therapy in diabetic retinopathy. Oxid. Med. Cell. Longev. 2017, 9702820 https://doi.org/10.1155/2017/9702820

Li S, Yang HW, Chen XL (2019): Protective effects of sulforaphane on diabetic retinopathy: activation of the Nrf2 pathway and inhibition of NLRP3 inflammasome formation. Exp. Anim. 68, 221-231 https://doi.org/10.1538/expanim.18-0146

Li Y, Shin D, Kwon SH (2013): Histone deacetylase 6 plays a role as a distinct regulator of diverse cellular processes. FEBS J. 280, 775-793 https://doi.org/10.1111/febs.12079

Lu L, Lu QY, Chen W, Li JW, Li CX, Zheng Z (2018): Vitamin D-3 Protects against diabetic retinopathy by inhibiting highglucose-induced activation of the ROS/TXNIP/NLRP3 inflammasome pathway. J. Diabetes Res. 2018, 8193523 https://doi.org/10.1155/2018/8193523

Lv PL, Yu JN, Xu XY, Lu TJ, Xu F (2019): Eriodictyol inhibits high glucose-induced oxidative stress and inflammation in retinal ganglial cells. J. Cell. Biochem. 120, 5644-5651 https://doi.org/10.1002/jcb.27848

Rangasamy S, McGuire PG, Franco Nitta C, Monickaraj F, Oruganti SR, Das A (2014): Chemokine mediated monocyte trafficking into the retina: role of inflammation in alteration of the bloodretinal barrier in diabetic retinopathy. PLoS One 9, e108508 https://doi.org/10.1371/journal.pone.0108508

Shi Q, Dong X, Zhang M, Cheng Y, Pei C (2019): Knockdown of ALK7 inhibits high glucose-induced oxidative stress and apoptosis in retinal pigment epithelial cells. Clin. Exp. Pharmacol. Physiol. 47, 313-321 https://doi.org/10.1111/1440-1681.13189

Shi Y, Zhang YZ, Li Y, Tong CJ (2019): Sauchinone inhibits high glucose-induced oxidative stress and apoptosis in retinal pigment epithelial cells. RSC Adv. 9, 17065-17071 https://doi.org/10.1039/C9RA02817J

Shi YF, Xu LQ, Tang JH, Fang L, Ma SC, Ma XY, Nie J, Pi XL, Qiu AD, Zhuang SG, Liu N (2017): Inhibition of HDAC6 protects against rhabdomyolysis-induced acute kidney injury. Am. J. Physiol. Renal. Physiol. 312, F502-F515 https://doi.org/10.1152/ajprenal.00546.2016

Willermain F, Scifo L, Weber C, Caspers L, Perret J, Delporte C (2018): Potential interplay between hyperosmolarity and inflammation on retinal pigmented epithelium in pathogenesis of diabetic retinopathy. Int. J. Mol. Sci. 19, 1056 https://doi.org/10.3390/ijms19041056

Wu Y, Tang L, Chen B (2014): Oxidative stress: implications for the development of diabetic retinopathy and antioxidant therapeutic perspectives. Oxid. Med. Cell. Longev. 2014, 752387 https://doi.org/10.1155/2014/752387
Xia T, Rizzolo LJ (2017): Effects of diabetic retinopathy on the barrier functions of the retinal pigment epithelium. Vision Res. 139, 72-81 https://doi.org/10.1016/j.visres.2017.02.006

Xia, Y, Shen S, Verma IM (2014): NF-kappaB, an active player in human cancers. Cancer Immunol. Res. 2, 823-830 https://doi.org/10.1158/2326-6066.CIR-14-0112

Xiao Q, Zhao Y, Xu J, Li WJ, Chen Y, Sun HJ (2019): NFE2/miR423-5p/TFF1 axis regulates high glucose-induced apoptosis in retinal pigment epithelial cells. BMC Mol. Cell. Biol. 20, 39 https://doi.org/10.1186/s12860-019-0223-2

Yi EH, Xu F, Li P, Guo JQ (2019): Transactive response DNA binding protein of 43/histone deacetylase 6 axis alleviates $\mathrm{H} 2 \mathrm{O} 2$-induced retinal ganglion cells injury through inhibiting apoptosis and autophagy. J. Cell. Biochem. 120, 4312-4320 https://doi.org/10.1002/jcb.27717

Yu XY, Liu QH, Wang XC, Liu H, Wang Y (2018): 7,8-Dihydroxyflavone ameliorates high-glucose induced diabetic apoptosis in human retinal pigment epithelial cells by activating TrkB. Biochem. Biophys. Res. Commun. 495, 922-927 https://doi.org/10.1016/j.bbrc.2017.11.007

Yu X, Luo Y, Chen G, Liu H, Tian N, Zen X, Liu Q (2019): Long noncoding RNA IGF2AS regulates high-glucose induced apoptosis in human retinal pigment epithelial cells. IUBMB Life 71, 1611-1618 https://doi.org/10.1002/iub.2102

Zhang WB, Yang F, Wang Y, Jiao FZ, Zhang HY, Wang LW, Gong ZJ (2019): Inhibition of HDAC6 attenuates LPS-induced inflammation in macrophages by regulating oxidative stress and suppressing the TLR4-MAPK/NF-kappa B pathways. Biomed. Pharmacother. 117, 9 https://doi.org/10.1016/j.biopha.2019.109166

Zhang X, Shi E, Yang L, Fu W, Hu F, Zhou X (2019): LncRNA AK077216 is downregulated in diabetic retinopathy and inhibited the apoptosis of retinal pigment epithelial cells by downregulating miR-383. Endocr. J. 66, 1011-1016 https://doi.org/10.1507/endocrj.EJ19-0080

Zhang X, Shi E, Yang L, Fu W, Hu F, Zhou X (2019): Gentiopicroside attenuates diabetic retinopathy by inhibiting inflammation, oxidative stress, and NF-kappa B activation in rat model. Eur. J. Inflamm. 17, 13 https://doi.org/10.1177/2058739219847837

Zhou Z, Liu J, Bi C, Chen L, Jiao Y, Cui L (2019): Knockdown of FOXO6 inhibits high glucose-induced oxidative stress and apoptosis in retinal pigment epithelial cells. J. Cell. Biochem. 120, 9716-9723 https://doi.org/10.1002/jcb.28252

Received: November 11, 2019

Final version accepted: December 3, 2019 